\title{
MicroRNA-496 suppresses tumor cell proliferation by targeting BDNF in osteosarcoma
}

\author{
JING YE $^{1}$, WEI XIE ${ }^{1}$, YUNZHOU ZUO $^{1}$, GUANGWU JING $^{2}$ and JIE TONG $^{2}$ \\ ${ }^{1}$ Department of Orthopedics, Hubei 672 Orthopedics Hospital of Integrated Chinese and Western Medicine, \\ Wuhan, Hubei 430079; ${ }^{2}$ Department of Orthopedics, The Sixth Hospital of Wuhan \\ Affiliated to Jianghan University, Wuhan, Hubei 430015, P.R. China
}

Received June 20, 2019; Accepted November 25, 2019

DOI: $10.3892 /$ etm.2019.8356

\begin{abstract}
MicroRNAs (miRNAs) are integrally involved in biological and pathobiological development. Many studies have demonstrated the abnormal expression of microRNA-496 (miR-496) in various human malignant tumors. The present study was designed to investigate the functions and the underlying mechanisms of miR-496 in osteosarcoma (OS) progression. Reverse transcription-quantitative polymerase chain reaction (RT-qPCR) was used to determine the expression of miR-496 in OS tissues and cell lines. Luciferase activity was used to confirm the interaction between miR-496 and brain derived neurotrophic factor $(B D N F)$, a downstream gene of miR-496. RT-qPCR was also used to quantify $B D N F$ mRNA expression, and the BDNF protein expression level was detected by western blot analysis. In addition, the Cell Counting Kit-8 (CCK-8) was used to detect cell viability. The results revealed that the level of miR-496 expression was significantly reduced in osteosarcoma tissues and cell lines. BDNF was verified to be a direct target gene of miR-496 and was found to be negatively regulated by miR-496. Overall, it was demonstrated that miR-496 inhibits osteosarcoma cell proliferation via inhibition of BDNF. Thus, the miR-496/BDNF axis may be a novel strategy for the clinical treatment of OS.
\end{abstract}

\section{Introduction}

Osteosarcoma (OS) is a type of malignant tumor commonly presenting in children, and adolescents (1) under 20 years of age (2). OS is caused by pathological changes in mesenchymal tissue in the bone, especially in the metaphysis of long bones (3). Osteosarcoma cells easily migrate to other tissues in a short period of time by the blood, and lungs are the main metastatic organ (4). With advanced chemotherapy

Correspondence to: Dr Jie Tong, Department of Orthopedics, The Sixth Hospital of Wuhan Affiliated to Jianghan University, 168 Hongkong Road, Wuhan, Hubei 430015, P.R. China

E-mail: casontong@163.com

Key word: osteosarcoma, miR-496, BDNF, proliferation techniques, the 5-year survival rate is approximately $60-70 \%$ in OS patients without distant metastasis (5). However, the 5 -year survival rate is lower than $30 \%$ for OS patients with distant metastasis (6). Thus, studies concerning the molecular mechanisms underlying OS carcinogenesis are vital for further clinical treatment.

MicroRNAs (miRNAs or miRs) are small non-coding RNAs consisting of 21-25 nucleotides (nt). miRNAs have been identified as key regulators of gene expression through binding with target mRNAs, thus affecting cell proliferation, division, apoptosis and metabolism (7-9). Dysregulation of miRNA expression is closely associated with various types of diseases and requires further research (10). Emerging research has revealed that miRNAs contribute to human carcinogenesis as tumor inhibitors or promoters. For example, miR-10a-5p was found to suppress human cervical cancer proliferation and division (11). Similarly, miR-214 plays a crucial role in melanoma tumor progression by targeting TFAP2C (12). miR-183 was found to contribute to advanced clinical stage human colorectal cancer (13). Moreover, miR-708 was demonstrated to suppress tumor cell proliferation in human glioblastoma (14). In order to investigate the relationship between miRNAs and tumors, we focused on the functions of miRNAs in OS progression.

miR-496 has been identified to participate in various pathobiological processes $(15,16)$. However, the functions of miR-496 in OS have not yet been elucidated. In the present study, expression of miR-496 was quantified in both OS tissues and cell lines. Brain derived neurotrophic factor $(B D N F)$ was identified one of the target genes of miR-496 in OS development. Based on these previous findings, the role of miR-496 and $B D N F$ in relation to the proliferation of OS cells was investigated.

\section{Materials and methods}

Tissue samples and cell lines. Pathological tissues and adjacent normal tissues were collected from 37 OS patients (22 male and 5 female; age, 17.23 \pm 7.56 years) during routine therapeutic surgery at the Department of Orthopedics, Hubei 672 Orthopedics Hospital of Integrated Chinese and Western Medicine (Wuhan, China) between June 2016 and October 2018. The patients had received no treatment at the 
time the tissues were extracted. Normal tissues were examined to confirm that there were no pathological changes. Liquid nitrogen was used to store the above tissues for the subsequent experiments. Written informed consent was obtained from all patients or their guardians, and the study protocol was approved by the Ethics Committee of Hubei 672 Orthopedics Hospital of Integrated Chinese and Western Medicine. The study was carried out in accordance with the approved guidelines.

OS cell lines. Based on the Enneking-Musculoskeletal Tumor Staging System (17), five types of OS cell lines, including hFOB1.19, MG-63, HOS, U2-OS and SAOS-2 were obtained from the Shanghai Cell Institute of Chinese Academy of Sciences (Shanghai, China). Dulbecco's modified Eagle's medium (DMEM; Gibco; Thermo Fisher Scientific, Inc.) was used to culture the cell lines. In addition, $10 \%$ fetal bovine serum (FBS) and $0.015 \mathrm{mg} / \mathrm{ml} \mathrm{5-bromo-2'-deoxyuridine}$ (Gibco; Thermo Fisher Scientific, Inc.) were added to the DMEM. The cells were incubated at $37^{\circ} \mathrm{C}$ in $5 \% \mathrm{CO}_{2}$.

Prediction of target genes of miR-496. Targetscan 3.1 (http://www.targetscan.org/mamm_31/) was used to determine the candidate downstream genes of miR-496. The prediction results and binding sites were obtained.

Reverse transcription-quantitative polymerase chain reaction (RT-qPCR). RNA was extracted using RNAiso Plus (Takara Biotechnology, Dalian, China). Prime Script RT Master Mix (Takara Biotechnology, Co., Ltd.) was used to reverse transcribe the RNA, and RT-qPCR was performed using SYBR Premix Ex Taq II (Takara Biotechnology). The following primers were used: GAPDH forward, 5'-GACTCATGACCA CAGTCCATGC-3' and GAPDH reverse, 5'-AGAGGCAGG GATGATGTTCTG-3'; BDNF forward, 5'-GGCTTGACATCA TTGGCTGAC-3' and BDNF reverse, 5'-CATTGGGCCGAA CTTTCTGGT-3'. miR-496 expression was quantified with the All-in-One ${ }^{\mathrm{TM}}$ miRNA qRT-PCR Detection Kit (GeneCopoeia, Inc., USA), in an Applied Biosystems 7900 Real-time PCR System (Thermo Fisher Scientific, Inc.).

Transfection. OS cells were transfected with miR-496 mimics (mimic-miR-496, 5'-CUCUAACCGGUACAUUA-3'), miR-496 inhibitors (inhibitor-miR-496, 5'-GAGAUUGGC CAUGUAAU-3') and their negative controls (mimic-NC, 5'-UUCUCCGAACGUGUCACGU-3' or inhibitor-NC, 5'-CAG UACUUUUGUGUAGUACAA-3'). LV-NC, LV-miR-496, or LV-miR-496+LV-BDNF were transfected into MG-63 and HOS cells, respectively. The lentiviruses used in our study were obtained from GenePharma (Shanghai, China). Lipofectamine $^{\circledR} 2000$ (Invitrogen; Thermo Fisher Scientific, Inc.) was used for cell transfection based on the manufacturer's protocols. Medium was replaced with fresh medium with $10 \%$ FBS after 6-h transfection.

Luciferase activity assay. Dual-Luciferase Reporter Assay System (Promega Corporation) was used to assay the activity of the luciferase reporter gene. The wild-type (BDNF 3'UTR ${ }^{\mathrm{WT}}$ ) or mutant type (BDNF 3'UTR ${ }^{\text {MUT }}$ ) binding to miR-496 was subcloned into the PGL3 Basic vector (Invitrogen; Thermo Fisher Scientific, Inc.). miR-496 mimics were co-transfected with $10 \mu \mathrm{g}$ of BDNF 3'UTR ${ }^{\mathrm{WT}}$ or BDNF 3'UTR ${ }^{\mathrm{MUT}}$ into MG-63 and HOS cells using Lipofectamine ${ }^{\circledR} 2000$ reagent, and cell medium was replaced with fresh medium with $10 \%$ FBS after $6 \mathrm{~h}$ transfection. The luciferase activity for each construct was normalized to that of the Renilla.

Western blot analysis. Proteins were obtained from the MG-63 and HOS cell lines and their concentration was estimated using the Bradford protein assay. The proteins (30 $\mu \mathrm{g} /$ lane) were separated by $10 \%$ sodium dodecyl sulfate-polyacrylamide gel (SDS-PAGE) and transferred to a polyvinylidene difluoride (PVDF) membrane. The membrane was blocked with $5 \%$ defatted milk at room temperature for $1 \mathrm{~h}$ and incubated with specific primary antibodies overnight with a controlled environment at $4^{\circ} \mathrm{C}$ overnight. After washing in TBST, the membranes were incubated with goat anti-rabbit horseradish peroxidase (HRP)-conjugated secondary antibodies (dilution 1:20,000, cat. no. ab205718; Abcam). Anti-BDNF (dilution 1:1,000, cat. no. ab108319; Abcam) and anti-glyceraldehyde-3-phosphate dehydrogenase (GAPDH) (dilution 1:10,000, cat. no. ab181602; Abcam) were used as the primary antibodies. Immunoreactive bands were developed using an enhanced chemiluminescence kit (cat. no. 170-5061; Bio-Rad Laboratories, Inc.) according to the manufacturer's instructions. Intensity of bands (BDNF, $15 \mathrm{kDa}$; GAPDH, $36 \mathrm{kDa}$ ) was quantified using ImageJ 1.49 (National Institutes of Health).

Cell viability assay. In order to evaluate the cell viability, CCK- 8 assay was employed to assess the cell growth and generate a growth curve. The transfected $5 \times 10^{3}$ OS cells were plated into a 96 -well plate and incubated at $37^{\circ} \mathrm{C}$ with $5 \% \mathrm{CO}_{2}$. Then, the cells were digested and counted every $24 \mathrm{~h}$. A cell growth curve was plotted after five days.

Statistical analysis. R Studio (3.5.1; https://www.r-project. org/) was performed for the statistical analyses. All data were from three independent experiments and were expressed as the mean \pm standard deviation (SD). Student paired t-tests were used for the comparison of miR-496 expression between primary tumors and adjacent noncancerous tissues. Student's unpaired t-test was used to measure the differences between quantitative variables. One-way analysis of variance (ANOVA) was used for the comparisons of the cell viability in the MG-63 $3^{\mathrm{LV}-\mathrm{NC}}$, MG-63 $3^{\mathrm{LV}-\mathrm{miR}-496}$, and MG-63 $3^{\mathrm{LV}-\mathrm{miR}-496+\mathrm{LV}-\mathrm{BDNF}}$ groups as well as in $\mathrm{HOS}^{\mathrm{LV}-\mathrm{NC}}$, $\mathrm{HOS}^{\mathrm{LV}-\mathrm{miR}-496}$, and $\mathrm{HOS}^{\mathrm{LV}-\mathrm{miR}-496+\mathrm{LV}-\mathrm{BDNF}}$. The post hoc Tukey's test was used to analyze the two-group comparisons. P-values $<0.05$ were considered statistically significant.

\section{Results}

Downregulation of miR-496 expression in OS tissues and cell lines. RT-qPCR was employed to determine miR-496 expression levels in osteosarcoma tissues and five OS cell lines. The results showed lower miR-496 expression level in the OS tissues than that in the normal control group (Fig. 1A). Moreover, miR-496 expression levels in OS MG-63, HOS, $\mathrm{U} 2-\mathrm{OS}$, and SAOS-2 cell lines were lower compared to the level in the hFOB1.19 cell line, which acted as a normal control 

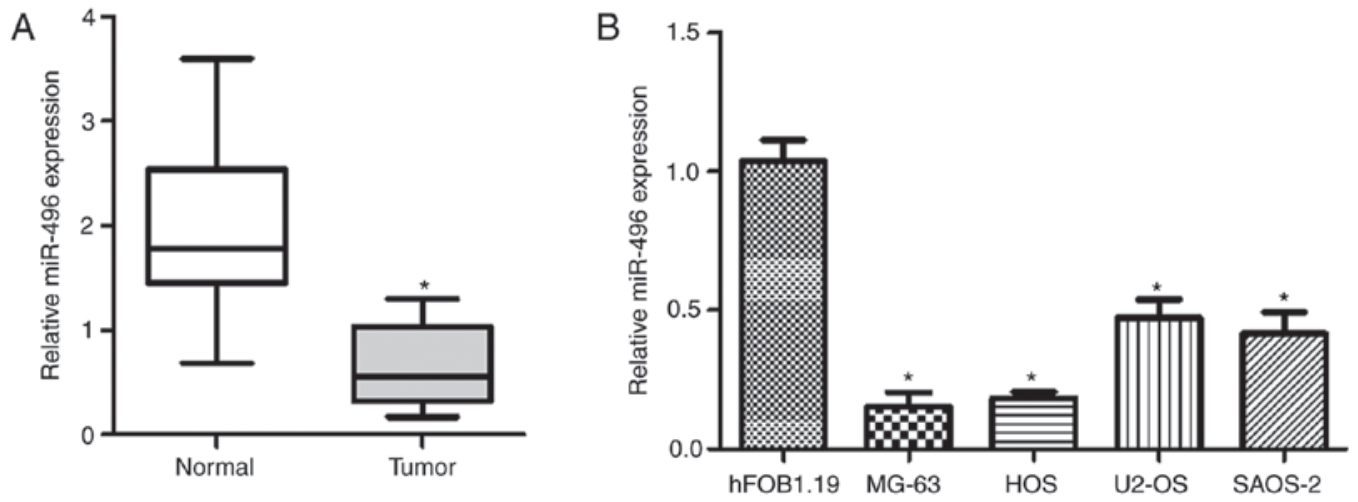

Figure 1. miR-496 expression is downregulated in OS tissues and cell lines. (A) miR-496 expression levels were compared between cancer tissues and paired adjacent normal tissues from 37 OS patients using RT-qPCR. (B) miR-496 expression in MG-63, HOS, U2-OS and SAOS-2 cell lines were significantly decreased compared with that noted in the control hFOB1.19 cell line. All experiments were performed three times. " $\mathrm{P}<0.05$, compared with the normal tissues or control hFOB1.19 cell line. OS, osteosarcoma.
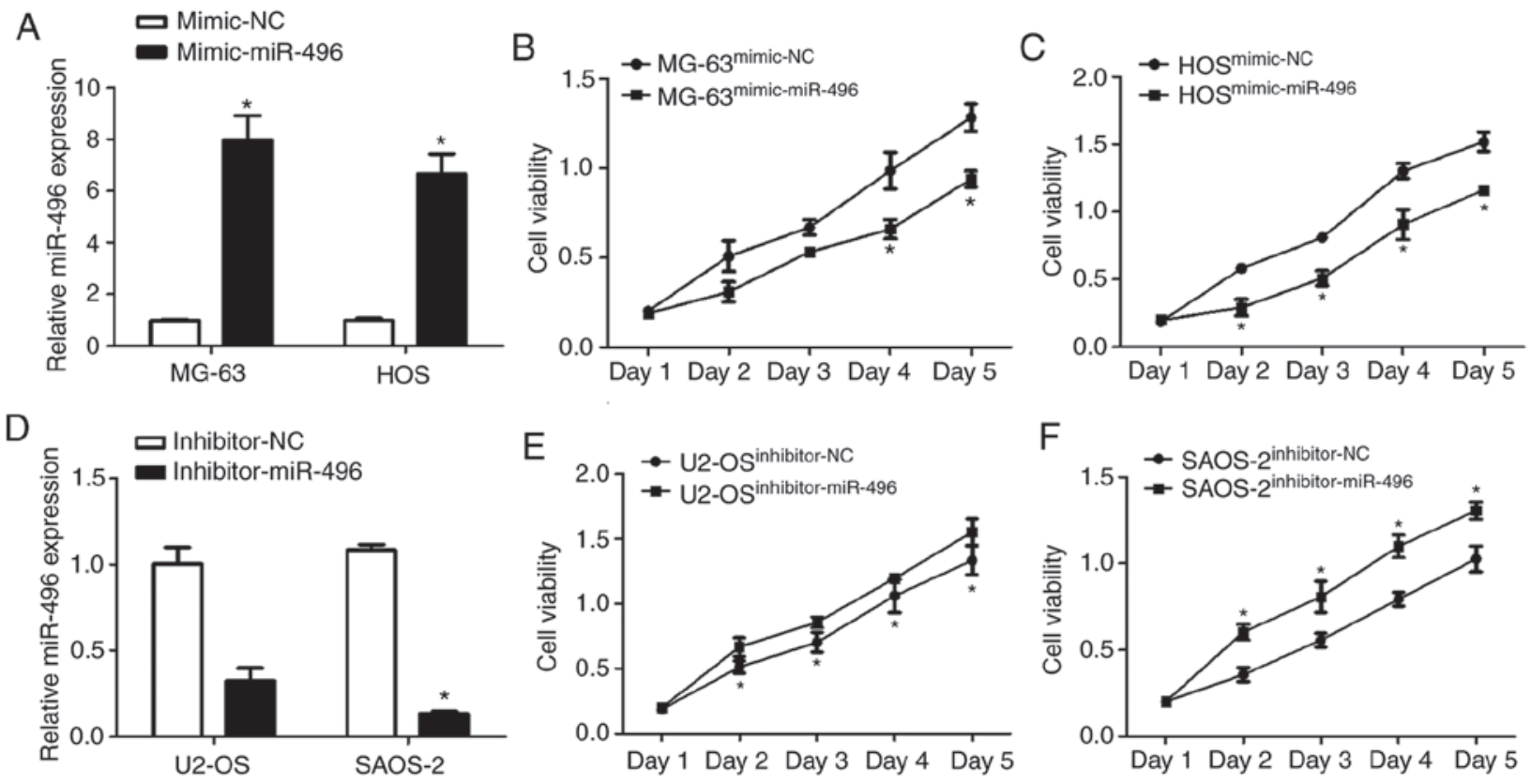

Figure 2. Overexpression of miR-496 reduces cell viability of OS MG-63 and HOS cells in vitro. (A) miR-496 was upregulated in OS MG-63 and HOS cell lines through transfection of miR-496 mimics. Cell viability was assessed by CCK-8 assay in (B) MG-63 and (C) HOS cell lines. (D) miR-496 was downregulated in U2-OS and SAOS-2 cell lines through transfection of miR-496 inhibitors. Cell viability was assessed by CCK-8 assay in (E) U2-OS and (F) SAOS-2 cell lines. All data were compared with the miR-496 mimic or inhibitor negative control (mimic-NC or inhibitor-NC); ${ }^{*} \mathrm{P}<0.05$. OS, osteosarcoma.

(Fig. 1B). The results showed that miR-496 contributes to OS development.

Effect of the altered expression of miR-496 on OS cell viability in vitro. In order to investigate the direct effect of miR-496 on cell viability, mimic-miR-496 and mimic-NC were transfected into MG-63 and HOS cells, and inhibitor-miR-496 and inhibitor-NC were transfected into U2-OS and SAOS-2 cells. miR-496 expression was increased in both MG-63 and HOS cell lines following the transfection of mimic-miR-496 (Fig. 2A). The cell viability of MG-63 cells transfected with the mimic-miR-496 was significantly decreased from day 4 compared to the mimic-NC-transfected cells (Fig. 2B). In addition, the cell viability of HOS cells transfected with the mimic-miR-496 was significantly decreased from Day 2 compared to mimic-NC-transfected cells (Fig. 2C).
In contrast, miR-496 expression was decreased in both U2-OS and SAOS-2 cell lines through the transfection of inhibitor-miR-496 (Fig. 2D). However, the cell viability of the U2-OS cells transfected with inhibitor-miR-496 was significantly increased compared to the inhibitor-NC-transfected cells (Fig. 2E). The cell viability of SAOS-2 cells transfected with inhibitor-miR-496 was also significantly decreased compared to the inhibitor-NC-transfected cells (Fig. 2F). These results indicate that miR-496 acts as a tumor suppressor in OS development.

$B D N F$ is a direct target of miR-496. Bioinformatic analysis was used to determine the downstream genes of miR-496 in OS progression, including ZIC1, CPEB2, CSMAD3, USD15, MYT1L, $S A T B 2$ and $E I F 4 B$. We found that BDNF 3'UTR could precisely bind to miR-496 (Fig. 3A). Furthermore, to verify whether BNDF 

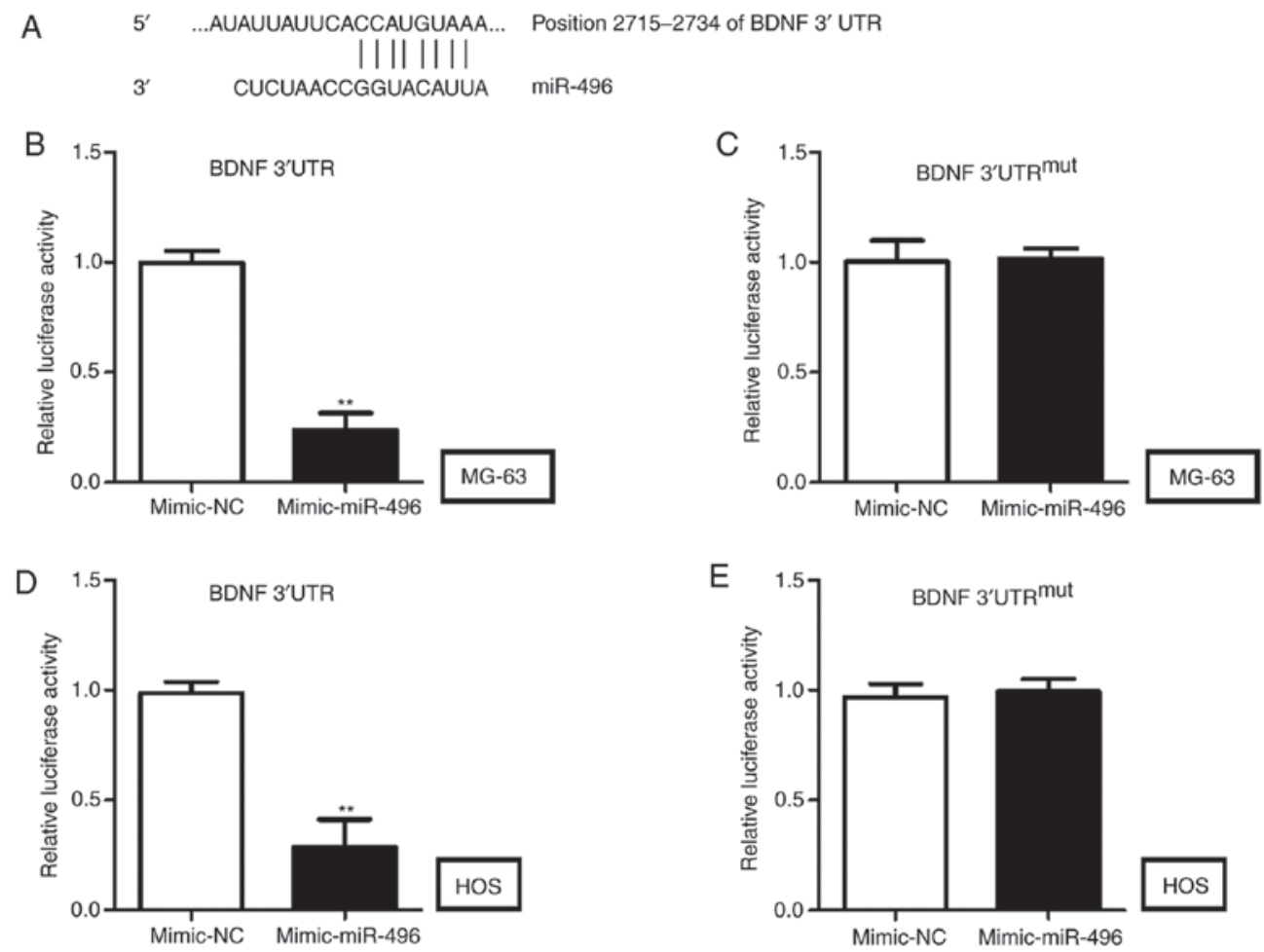

Figure 3. BDNF is a direct target gene of miR-496 in OS. (A) The binding site of miR-496 and BDNF 3' UTR. (B) MG-63 cells were co-transfected with miR-496 mimics or their negative control (mimic-NC) and MT-BDNF 3'UTR. The dual-luciferase vector was employed to detect relative luciferase activity in the MG-63 cells. (C) MG-63 cells were co-transfected with miR-496 mimics or their negative control and MUT-BDNF 3'UTR. (D) HOS cells were co-transfected with miR-496 mimics or their negative control and MT-BDNF 3'UTR. (E) HOS cells were co-transfected with miR-496 mimics or their negative control and MUT-BDNF 3'UTR. . * P $<0.01$. BDNF, brain derived neurotrophic factor; OS, osteosarcoma; UTR, untranslated region.
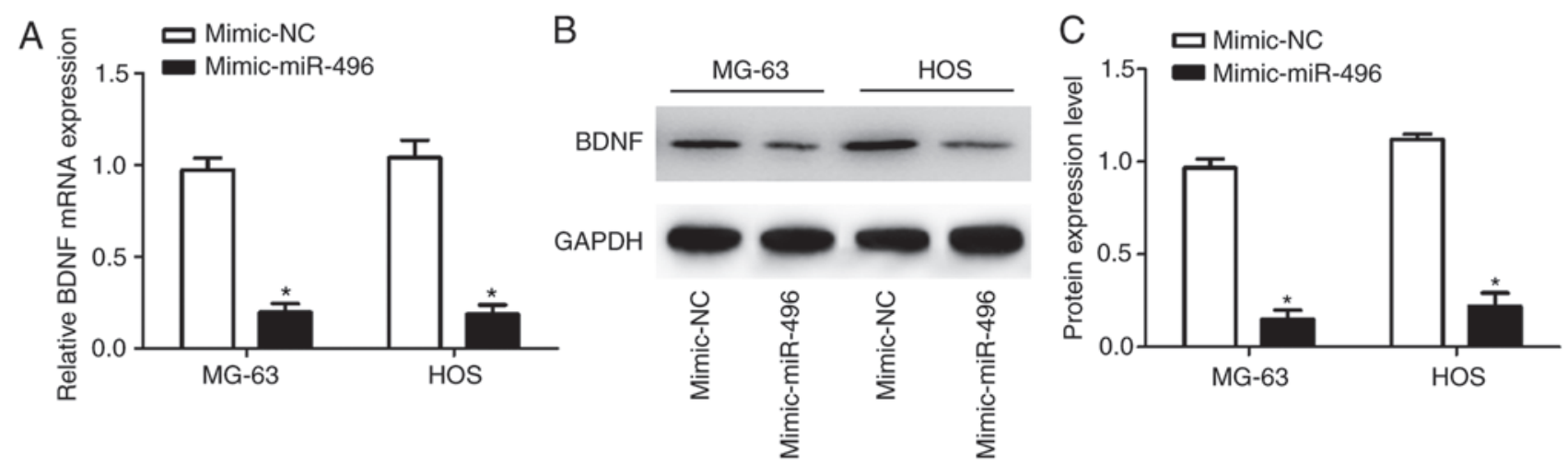

Figure 4. Overexpression of miR-496 suppressed BDNF expression in OS MG-63 and HOS cell lines. (A) mRNA expression of BDNF in MG-63 and HOS cells transfected with miR-496 mimics (mimic-miR-496) or their negative control (mimic-NC), respectively. (B and C) Effect of miR-496 on BDNF protein levels in MG-63 and HOS cells. "P<0.05, compared with the mimic-NC group. BDNF, brain derived neurotrophic factor; OS, osteosarcoma.

is a direct target gene, wild-type (WT) or mutant (MUT) 3'UTR of BDNF was transfected into the luciferase vector. The vector co-transfected with miR-496 mimics and BDNF $3^{\prime} \mathrm{UTR}^{\mathrm{WT}}$ had lower luciferase activity in both MG-63 (Fig. 3B) and HOS cells (Fig. 3D). However, co-transfection of miR-496 mimics with the luciferase vector containing MUT-BDNF 3'UTR had the same luciferase activity compared to the mimic negative control in both MG-63 (Fig. 3C) and HOS cells (Fig. 3E). This demonstrated that $B D N F$ is a downstream gene of miR- 496 .

miR-496 and BDNF expression in OS. In order to determine the function of miR-496 in OS, RT-qPCR and western blot analysis were used to measure BDNF mRNA and protein expression levels. Overexpression of miR-496 suppressed BDNF in both MG-63 and HOS cells at the mRNA (Fig. 4A) and protein (Fig. 4B and C) levels. This finding thus confirms that miR-496 negatively modulates BDNF expression.

Overexpression of BDNF in OS. BDNF protein expression and cell viability were assessed to investigate the function of BDNF in OS through BDNF upregulation in MG-63 and HOS cells. The BDNF protein expression level was increased in both MG-63 (Fig. 5A and B) and HOS cells (Fig. 5C and D) following co-transfection of LV-miR-496+LV-BDNF, compared to the 
A

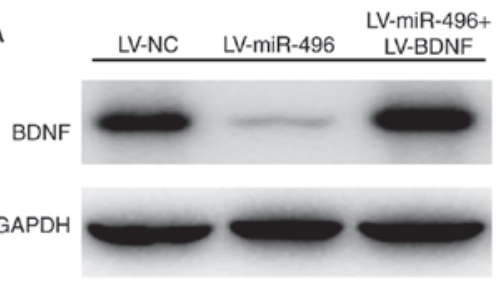

B

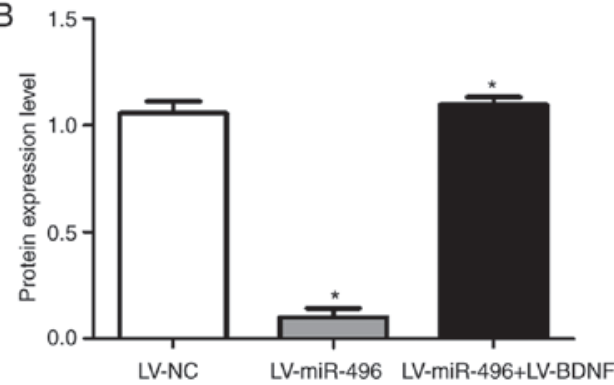

C

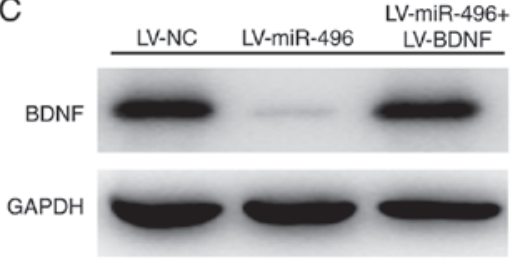

D

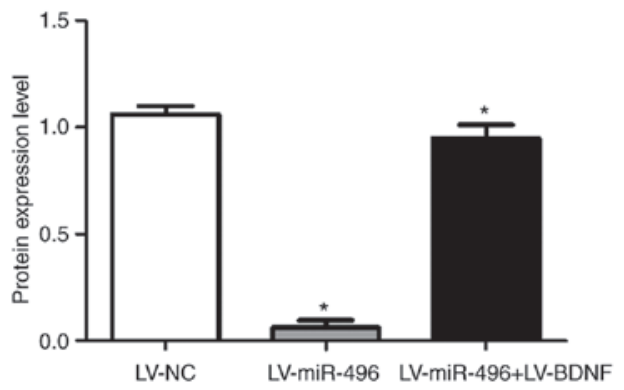

Figure 5. BDNF expression is modulated by miR-496 in vitro. BDNF protein expression levels in OS (A and B) MG-63 and (C and D) HOS cells. LV-miR-496 or LV-miR-496+LV-BDNF were transfected into MG-63 and HOS cells and the protein expression of BNDF was assessed. LV-NC acted as the control group. ${ }^{*} \mathrm{P}<0.05$, compared with the LV-NC group. BDNF, brain derived neurotrophic factor; OS, osteosarcoma.

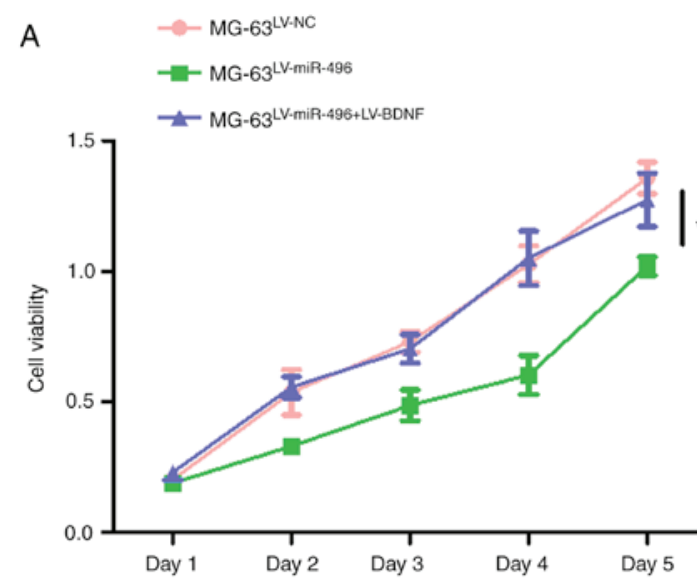

C
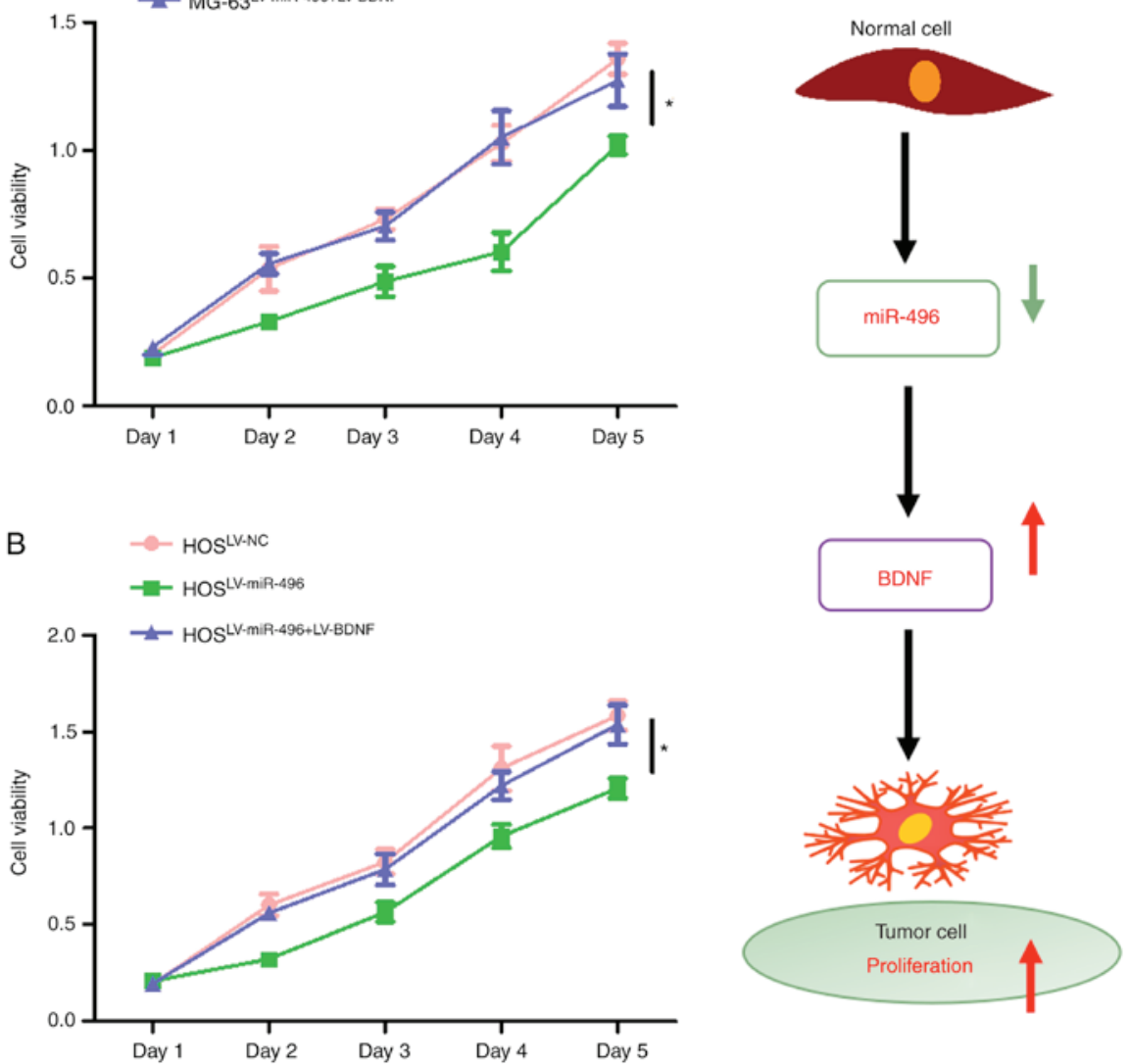

Figure 6. Overexpression of BDNF promotes cell proliferation of OS MG-63 and HOS cell lines. Cell viability was measured by CCK-8 assay in (A) MG-63 and (B) HOS cells. LV-miR-496 or LV-miR-496+LV-BDNF were transfected into MG-63 and HOS cells. LV-NC acted as the control group. "P<0.05. (C) miR-496 expression is downregulated in OS tissues. $B D N F$ is directly targeted by miR-496. BDNF thus acts as a promoter of OS progression. Thus, the miR-496/BDNF axis provides a novel therapeutic target for OS. $B D N F$, brain derived neurotrophic factor; OS, osteosarcoma.

transfection with LV-miR-496 only. Moreover, cell viability was increased in both MG-63 (Fig. 6A) and HOS cells (Fig. 6B) following LV-miR-496+LV-BDNF co-transfection.
This indicated that overexpression of BDNF reversed the suppressive effect of miR-496 on OS cell viability (Fig. 6C). BDNF thus acts as a promoter of OS progression. 


\section{Discussion}

Several studies have identified microRNAs as oncogenes or tumor suppressors in various types of cancer, including human osteosarcoma (OS) (18-20). For example, miR-181a was found to promote cell proliferation and invasion in OS (21). miR-504 was found to induce cell proliferation and suppress apoptosis as well as G1 phase arrest in OS via negatively regulating p53 (22). Moreover, miR-758 suppressed the malignant phenotype of OS cells by directly targeting HMGA1 (23). miR-214 was found to accelerate the progression of OS by modulating the Wnt/ $\beta$-catenin signaling pathway (24). miR-143-3p was demonstrated to inhibit the proliferation and migration in OS by targeting FOSL2 (25). The different mechanisms of miRNAs in OS provide a possible orientation for the clinical diagnosis and treatment of OS (26). Therefore, comprehensive research is needed to explore the targets of miRNAs in the clinical therapy of OS; specifically drawing our attention to miR-496.

Previous studies have indicated that miR-496 is associated with several human pathological changes, including oropharyngeal cancer (27), cerebral ischemia/reperfusion injury (28), type 2 diabetes mellitus and obesity (29), and osteogenesis of human bone marrow (30). These findings suggest that miR-496 serves as a suppressor in human pathological changes, which is consistent with our findings. In the present study, miR-496 expression was downregulated in OS cell lines. Overexpression of miR-496 reduced cell viability in both MG-63 and HOS cell lines, and loss of miR-496 promoted cell viability in both U2-OS and SAOS-2 cell lines. To note, miR-496 expression in the MG-63 and HOS cell lines was the lowest, which is the main reason why the MG-63 and HOS cell lines were chosen to perform the functional experiments.

Brain-derived neurotrophic factor $(B D N F)$ gene is related to nervous system development (31). Previous research has shown that BDNF contributes to cancer development. For example, the BDNF/leptin axis was correlated with cancer remission and inhibition (32). BDNF was found to act as an inhibitor in breast cancer development and metastasis in mice (33). miR-10a-5p was found to suppress human cervical cancer progression by targeting BDNF (11). In the present study, BDNF was proposed as the target gene of miR-496 in OS progression. We found that miR-496 negatively modulated BDNF expression in OS cells. Overexpression of BDNF promoted cell proliferation of the OS cells. This indicated that BDNF is a promoter of OS development.

Recently, a similar study revealed that overexpression of miR-496 suppressed human OS cell proliferation, invasion, and migration by targeting eIF4E (34). These previous results reinforced our finding that miR-496 acts as a suppressor of human OS cell proliferation.

To conclude, miR-496 expression was downregulated in OS tissues and cell lines, whereas the overexpression of miR-496 reduced cell viability and suppressed cell proliferation in OS cell lines. BDNF is directly targeted by miR-496. Upregulation of BDNF promoted cell proliferation in the OS cell lines. Thus, the miR-496/BDNF axis provides a novel therapeutic target for OS (Fig. 6C). miR-496 may be one of the epigenetic modifications to regulate the expression of BDNF. Other mechanisms such as DNA methylation, histone modifications, kinase-associated phosphorylation may also affect BDNF expression. A larger sample size with more pathological information and functional studies with a focus on cell apoptosis, cell migration and cell invasion as well as in vivo experiments are necessary to further confirm our results.

\section{Acknowledgements}

Not applicable.

\section{Funding}

No funding was received.

\section{Availability of data and materials}

All data generated or analyzed during this study are included in this published article.

\section{Authors' contributions}

JT conceived and designed the study. JY, WX, YZ and GJ performed the experiments. JY and JT wrote the paper. All authors read and approved the manuscript and agree to be accountable for all aspects of the research in ensuring that the accuracy or integrity of any part of the work are appropriately investigated and resolved.

\section{Ethics approval and consent to participate}

Written informed consent was obtained from all patients or their guardians, and the study protocol was approved by the Ethics Committee of Hubei 672 Orthopedics Hospital of Integrated Chinese and Western Medicine.

\section{Patient consent for publication}

Not applicable.

\section{Competing interests}

The authors declare that they have no competing interest.

\section{References}

1. Yang J and Zhang W: New molecular insights into osteosarcoma targeted therapy. Curr Opin Oncol 25: 398-406, 2013.

2. Yu W, Zhu J, Wang Y, Wang J, Fang W, Xia K, Shao J, Wu M, Liu B, Liang C, et al: A review and outlook in the treatment of osteosarcoma and other deep tumors with photodynamic therapy: From basic to deep. Oncotarget 8: 39833-39848, 2017.

3. Berner K, Johannesen TB, Berner A, Haugland HK, Bjerkehagen B, Bøhler PJ and Bruland OS: Time-trends on incidence and survival in a nationwide and unselected cohort of patients with skeletal osteosarcoma. Acta Oncol 54: 25-33, 2015.

4. Taran SJ, Taran R and Malipatil NB: Pediatric osteosarcoma: An updated review. Indian J Med Paediatr Oncol 38: 33-43, 2017.

5. Faisham WI, Mat Saad AZ, Alsaigh LN, Nor Azman MZ, Kamarul Imran M, Biswal BM, Bhavaraju VM, Salzihan MS, Hasnan J, Ezane AM, et al: Prognostic factors and survival rate of osteosarcoma: A single-institution study. Asia Pac J Clin Oncol 13: e104-e110, 2017. 
6. Wang S, Zheng S, Hu K, Sun H, Zhang J, Rong G, Gao J, Ding N and Gui B: A predictive model to estimate the pretest probability of metastasis in patients with osteosarcoma. Medicine (Baltimore) 96: e5909, 2017.

7. Bartel DP: MicroRNAs: Target recognition and regulatory functions. Cell 136: 215-233, 2009.

8. Sonkoly E: The expanding microRNA world in psoriasis. Exp Dermatol 26: 375-376, 2017.

9. Mellis D and Caporali A: MicroRNA-based therapeutics in cardiovascular disease: Screening and delivery to the target. Biochem Soc Trans 46: 11-21, 2018

10. Acunzo M and Croce CM: MicroRNA in cancer and cachexia-A mini-review. J Infect Dis 212 (Suppl 1): S74-S77, 2015.

11. Zhai L, Li Y, Lan X and Ai L: MicroRNA-10a-5p suppresses cancer proliferation and division in human cervical cancer by targeting BDNF. Exp Ther Med 14: 6147-6151, 2017.

12. Penna E, Orso F, Cimino D, Tenaglia E, Lembo A, Quaglino E, Poliseno L, Haimovic A, Osella-Abate $\mathrm{S}$, De Pittà C, et al: microRNA-214 contributes to melanoma tumour progression through suppression of TFAP2C. EMBO J 30: 1990-2007, 2011.

13. Zhou T, Zhang GJ, Zhou H, Xiao HX and Li Y: Overexpression of microRNA-183 in human colorectal cancer and its clinical significance. Eur J Gastroenterol Hepatol 26: 229-233, 2014.

14. Guo P, Lan J, Ge J, Nie Q, Mao Q and Qiu Y: miR-708 acts as a tumor suppressor in human glioblastoma cells. Oncol Rep 30 870-876, 2013

15. Rubie C, Kölsch K, Halajda B, Eichler H, Wagenpfeil S, Roemer K and Glanemann M: microRNA-496-A new, potentially aging-relevant regulator of mTOR. Cell Cycle 15: 1108-1116, 2016.

16. Hu J, Liu T, Zhang Z, Xu Y and Zhu F: Oxidized low-density lipoprotein promotes vascular endothelial cell dysfunction by stimulating miR-496 expression and inhibiting the Hippo pathway effector YAP. Cell Biol Int 43: 528-538, 2019.

17. Cates JMM: Simple staging system for osteosarcoma performs equivalently to the AJCC and MSTS systems. J Orthop Res 36: 2802-2808, 2018.

18. Di Leva G, Garofalo M and Croce CM: MicroRNAs in cancer. Annu Rev Pathol 9: 287-314, 2014.

19. Acunzo M, Romano G, Wernicke D and Croce CM: MicroRNA and cancer-a brief overview. Adv Biol Regul 57: 1-9, 2015.

20. Palmini G, Marini F and Brandi ML: What is new in the miRNA world regarding osteosarcoma and chondrosarcoma? Molecules 22: pii: E417, 2017.

21. Jianwei Z, Fan L, Xiancheng L, Enzhong B, Shuai L and Can L: MicroRNA 181a improves proliferation and invasion, suppresses apoptosis of osteosarcoma cell. Tumour Biol 34: 3331-3337, 2013

22. Chen X, Lv C, Zhu X, Lin W, Wang L, Huang Z, Yang S and Sun J: MicroRNA-504 modulates osteosarcoma cell chemoresistance to cisplatin by targeting p53. Oncol Lett 17: 1664-1674, 2019.
23. Ren J, Yang M, Xu F and Chen J: microRNA-758 inhibits the malignant phenotype of osteosarcoma cells by directly targeting HMGA1 and deactivating the Wnt/ $\beta$-catenin pathway. Am J Cancer Res 9: 36-52, 2019.

24. Zhu XB, Zhang ZC, Han GS, Han JZ and Qiu DP: Overexpression of miR-214 promotes the progression of human osteosarcoma by regulating the Wnt $/ \beta$-catenin signaling pathway. Mol Med Rep 15: 1884-1892, 2017.

25. Sun X, Dai G, Yu L, Hu Q, Chen J and Guo W: miR-143-3p inhibits the proliferation, migration and invasion in osteosarcoma by targeting FOSL2. Sci Rep 8: 606, 2018.

26. Miao J, Wu S, Peng Z, Tania M and Zhang C: MicroRNAs in osteosarcoma: Diagnostic and therapeutic aspects. Tumour Biol 34: 2093-2098, 2013.

27. Mason D, Zhang X, Marques TM, Rose B, Khoury S, Hill M, Deutsch F, Lyons JG, Gama-Carvalho M and Tran N: Human papillomavirus 16 E6 modulates the expression of miR-496 in oropharyngeal cancer. Virology 521: 149-157, 2018.

28. Yao X, Yao R, Yi J and Huang F: Upregulation of miR-496 decreases cerebral ischemia/reperfusion injury by negatively regulating BCL2L14. Neurosci Lett 696: 197-205, 2019.

29. Rubie C, Zimmer J, Lammert F, Gross JC, Weber SN, Kruse B, Halajda B, Wagner M, Wagenpfeil S and Glanemann M: MicroRNA-496 and mechanistic target of rapamycin expression are associated with type 2 diabetes mellitus and obesity in elderly people. Ann Nutr Metab 74: 279-286, 2019.

30. Huang $\mathrm{J}$ and Chen L: IL-1 $\beta$ inhibits osteogenesis of human bone marrow-derived mesenchymal stem cells by activating FoxD3/microRNA-496 to repress wnt signaling. Genesis 55, 2017.

31. Mariga A,Mitre M and Chao MV: Consequences of brain-derived neurotrophic factor withdrawal in CNS neurons and implications in disease. Neurobiol Dis 97: 73-79, 2017.

32. Cao L, Liu X, Lin EJ, Wang C, Choi EY, Riban V, Lin B and During MJ: Environmental and genetic activation of a brain-adipocyte BDNF/leptin axis causes cancer remission and inhibition. Cell 142: 52-64, 2010.

33. Liu X, McMurphy T, Xiao R, Slater A, Huang W and Cao L: Hypothalamic gene transfer of BDNF inhibits breast cancer progression and metastasis in middle age obese mice. Mol Ther 22: 1275-1284, 2014.

34. Qi NN, Tian S, Li X, Wang FL and Liu B: Up-regulation of microRNA-496 suppresses proliferation, invasion, migration and in vivo tumorigenicity of human osteosarcoma cells by targeting eIF4E. Biochimie 163: 1-11, 2019. 